\title{
A multiscale procedure based on the stochastic kinetic mean field and the phase- field models for coarsening
}

Cite as: J. Appl. Phys. 126, 065106 (2019); https://doi.org/10.1063/1.5099676

Submitted: 12 April 2019 . Accepted: 20 July 2019. Published Online: 13 August 2019

Bence Gajdics, János J. Tomán (iD, Helena Zapolsky, Zoltán Erdélyi, and Gilles Demange

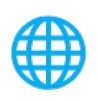

\section{ARTICLES YOU MAY BE INTERESTED IN}

Growth of graphitic carbon layers around silicon carbide nanowires

Journal of Applied Physics 126, 065304 (2019); https://doi.org/10.1063/1.5098987

Spindle-like $\mathrm{Fe}_{3} \mathrm{O}_{4}$ nanoparticles for improving sensitivity and repeatability of giant magnetoresistance biosensors

Journal of Applied Physics 126, 064505 (2019); https://doi.org/10.1063/1.5096345

Theoretical prediction and experimental demonstration of inter-valley scattering induced Inverse Spin Hall effect for hot electrons in GaAs

Journal of Applied Physics 126, 065703 (2019); https://doi.org/10.1063/1.5108860

\section{Journal of Applied Physics Polymer-Grafted Nanoparticles}




\title{
A multiscale procedure based on the stochastic kinetic mean field and the phase-field models for coarsening
}

\author{
Cite as: J. Appl. Phys. 126, 065106 (2019); doi: 10.1063/1.5099676 \\ Submitted: 12 April 2019 . Accepted: 20 July 2019 . \\ Published Online: 13 August 2019
}

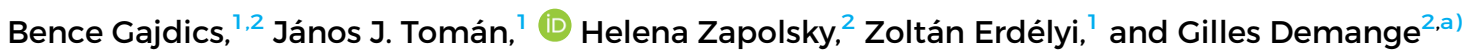

\author{
AFFILIATIONS \\ ${ }^{7}$ Department of Solid State Physics, University of Debrecen, P.O. Box 400, H-4002 Debrecen, Hungary \\ ${ }^{2}$ GPM, UMR CNRS 6643, University of Rouen, 76575 Saint Étienne du Rouvray, France \\ a) gilles.demange@univ-rouen.fr
}

\begin{abstract}
We report a quantitative multiscale (MS) procedure based on the recently developed Stochastic Kinetic Mean Field approach (SKMF) [Erdélyi, M. Pasichnyy, V. Bezpalchuk, J. J. Tomán, B. Gajdics, and A. M. Gusak, Comput. Phys. Commun. 204, 31-37 (2016)], combined with the Phase Field model (PFM) and CALPHAD database, to study the nucleation-growth-coarsening process in alloys. The SKMF approach reproduces the nucleation and early growth of precipitates in the matrix, and the PFM then simulates the coarsening of the microstructure. To ensure the consistency of the procedure, the length and time scales of SKMF and PFM are explicitly connected. Moreover, both the effective interaction energies used in the SKMF and the free energy used in the PFM are taken from CALPHAD database. Two different implementations of the procedure are proposed. First, the postnucleation microstructure as provided by SKMF is used as the initial condition for subsequent PFM simulations. Second, only the particle size distribution and particle density are transferred to PFM, thereby giving access to bigger systems. The proposed procedure is tested in the specific case of the Ag-Cu model alloy.
\end{abstract}

Published under license by AIP Publishing. https://doi.org/10.1063/1.5099676

\section{INTRODUCTION}

The development of new efficient multiscale (MS) approaches has recently become a first class issue in the field of materials science. Indeed, only MS models can comprehensively account for the numerous dynamical processes rooting the formation of complex microstructures, including structural phase transitions, precipitation, grain boundary formation, or defect kinetics. ${ }^{1,2}$ In particular, the dynamics of the nucleation-growth-coarsening process has specifically sparked the development of a series of MS approaches. $^{3-6}$ These involve atomistic models downscale, such as Molecular Dynamics (MD) ${ }^{7}$ and Kinetic Monte Carlo (KMC), ${ }^{8}$ and mesoscopic approaches upscale such as the Phase Field Model (PFM). ${ }^{9-11}$ PFM was notably proved efficient to simulate coarsening kinetics in multicomponent alloys, first qualitatively ${ }^{12}$ and then quantitatively when combined with CALPHAD data, which provides the thermodynamic description of systems. ${ }^{13}$ The founding principle of PFM based MS procedures is to evaluate the phenomenological parameters stepping in the PFM equations, by means of various smaller scale approaches. ${ }^{3-5,14}$ This paragon was then improved by using KMC simulations of the postnucleation microstructure as the initial condition for the subsequent PFM calculations. ${ }^{6}$

However, these MS procedures can be hard to reproduce, notably because the integrated numerical approaches are manifold and sometimes not entirely transferable to other materials. ${ }^{6}$ For this reason, we propose a simple alternative MS approach in this work, where the recently developed Stochastic Kinetic Mean Field (SKMF) model ${ }^{15,16}$ is used on the atomic scale, whereas PFM is used for the longer scale. SKMF was adapted from the Kinetic Mean Field (KMF) model ${ }^{17}$ generalized from the original 1D model of George Martin, ${ }^{18}$ to which a dynamic Langevin noise was added as it provides results similar to the lattice KMC. ${ }^{19}$ However, the connection between SKMF and PFM is easier, as the SKMF internal energy involves composition dependent effective interaction energies, which can be taken from CALPHAD, thereby, it can match the chemical free energy used in the PFM. This connection is justified by the equivalence of the stationary states of the SKMF on the atomic scale, and the local minima of the Mean-Field Bragg-Williams (MFBW) free energy on the mesoscale. ${ }^{18,20,21}$ 
Another insight of the proposed MS procedure is to explicitly match the space and time scales between atomic (SKMF) and mesoscopic (PFM) levels. By doing so, a consistent integration of the constitutive models within the MS procedure can be achieved.'

The proposed model is tested on the silver-copper binary alloy, notably because it undergoes an isostructural precipitation when the binodal is crossed. Besides, silver-copper alloys are still considered for microelectronics applications. ${ }^{22}$

This work is organized as follows. First, a updated version of the SKMF approach accounting for the more general case of asymmetric phase diagrams is presented. Then, the PFM is briefly refreshed. To parametrize the proposed MS procedure based on SKMF and PFM, the space and time scales of the two constituting models are connected by matching the interface profiles and growth velocity of an isolated growing precipitate, in the case study of the $\mathrm{AgCu}$ model alloy. For both approaches, the thermodynamic data are taken from CALPHAD. Therefrom, the MS procedure is implemented to simulate the full nucleation-growth-coarsening process. Two strategies are proposed. First, the PFM is fed with the SKMF simulations of the microstructure. Second, the MS procedure is upscaled, as the particle size distribution (PSD) and the number of precipitates provided by SKMF are used as the input for the PFM simulations.

\section{NUMERICAL METHOD}

\section{A. Stochastic kinetic mean-field model}

The SKMF approach ${ }^{15,16}$ is based on the model of Martin. ${ }^{18}$ Here, the local atomic configuration in a A-B binary alloy is described by the occupation probability $c_{i}$ of $\mathrm{A}$ atoms on a site $i$ so that $1-c_{i}$ is the occupation probability of $B$ atoms on the same site. The time evolution of $c_{i}$ is governed by a nonlinear stochastic equation satisfying the balance of the conservation of matter and the fluxes of atoms between the site $i$ and its $Z$ nearest neighboring sites $j$,

$$
\frac{\mathrm{d} c_{i}}{\mathrm{~d} t}=\sum_{j=1}^{Z}\left[J_{j \rightarrow i}-J_{i \rightarrow j}\right] .
$$

In this equation, $J_{j \rightarrow i}$ and $J_{i \rightarrow j}$ are the material fluxes of $\mathrm{A}$ atoms from site $j$ to site $i$ and vice versa, both containing a mean-field and a fluctuation part,

$$
J_{i \rightarrow j}=J_{i \rightarrow j}^{\mathrm{MF}}+\delta J_{i \rightarrow j}^{\mathrm{Lang}} .
$$

Here, $J_{i \rightarrow j}^{\mathrm{MF}}$ is the mean-field (MF) atomic flux from site $i$ to site $j$, defined from the mean-field (MF) exchange rate $\Gamma_{i, j}^{\mathrm{MF}}$ between sites $i$ and $j$ as follows:

$$
J_{i \rightarrow j}^{\mathrm{MF}}=c_{i}\left(1-c_{j}\right) \Gamma_{i, j}^{\mathrm{MF}}
$$

Similarly, the fluctuation part $\delta J_{i \rightarrow j}^{\mathrm{Lang}}$ in the total flux can be expressed from a dynamic Langevin noise $\delta_{i, j}^{\text {Lang }}$ as $^{15}$

$$
\delta J_{i \rightarrow j}^{\mathrm{Lang}}=c_{i}\left(1-c_{j}\right) \delta_{i, j}^{\mathrm{Lang}},
$$

where the dynamic Langevin noise $\delta_{i, j}^{\mathrm{Lang}}$ is but a white noise of amplitude $A_{n}$

$$
\delta_{i, j}^{\mathrm{Lang}}=\frac{A_{n}}{\sqrt{\Delta t}} \theta
$$

where $\Delta t$ is the time step of the simulation. In compliance with the definition of a Gaussian noise, $\theta$ is a random uniform number of zero mean value and a mean squared value equal to 1 . The introduction of stochasticity in the SKMF model makes it possible to describe the initial stages of nucleations, thereby overcoming one limitation of the deterministic model of Martin. It should be noted that adding the noise to the flux rather than the concentration notably allows to circumvent potential singularities in the composition change rates. ${ }^{15}$ When $A_{n}$ is set to zero, the SKMF model becomes purely deterministic.

The MF jump frequency $\Gamma_{i, j}^{\mathrm{MF}}$ is defined as

$$
\Gamma_{i, j}^{\mathrm{MF}}=v \exp \left(-\frac{E_{0}-\left(E_{i}^{A}+E_{j}^{B}\right)}{k_{b} T}\right) .
$$

Here, $k_{b}$ is the constant of Boltzmann, $T$ is the absolute temperature, and $v$ is the frequency of the atoms trying to reach the saddle point energy $E_{0}$ assumed constant in this work. Besides, each energy $E_{s}^{X}(X=A, B ; s=i, j)$ is the sum of the interaction energies between an atom $\mathrm{X}$ on site $s$ and the $Z$ nearest neighbor atoms in the vicinity $\mathcal{V}(s)$ of site $s$ in the first coordination shell,

$$
E_{s}^{X}=\sum_{l=1, l \in \mathcal{V}(s)}^{Z}\left[c_{l} V_{A X}+\left(1-c_{l}\right) V_{X B}\right]
$$

where $V_{A A}, V_{B B}$, and $V_{A B}$ are the $A-A, B-B$, and $A-B$ pair interaction energies in the $A-B$ alloy, respectively. It should be noted that a more realistic description of a system could be provided by the $n$-body interaction model, as it might lead to a more accurate estimation of jump frequencies and interface energies. The SKMF model is not restricted to pair-interaction energies. It can be equipped with $n$-body potentials instead, and atomic interactions beyond the first coordination shell can also be considered. We stick to the pair interactions for demonstration purposes in the case of the $\mathrm{Ag}-\mathrm{Cu}$ system. An equivalent expression of the MF jump frequency $\Gamma_{i, j}^{\mathrm{MF}}$ is used in practice in SKMF simulations, on introducing the parameters,

$$
\mathcal{M}=\frac{1}{2}\left(V_{A A}-V_{B B}\right), \quad V=\frac{1}{2}\left(V_{A B}-\left[V_{A A}+V_{B B}\right]\right) .
$$

The parameter $V$ corresponds to the mixing energy of the solid solution, and the parameter $\mathcal{M}$ accounts for the A/B asymmetry of the tracer diffusion coefficients. Thereby, different values for the parameter $\mathcal{M}$ will result in different kinetic pathways toward equilibrium (e.g., interface sharpening instead of broadening in miscible alloys $\left.{ }^{23,24}\right)$. Then, $\Gamma_{i, j}^{\mathrm{MF}}$ can customarily be rewritten 
in the alternative form

$$
\Gamma_{i, j}^{\mathrm{MF}}=\Gamma_{0} \exp \left(-\frac{\widehat{E}_{i, j}}{k_{b} T}\right)
$$

where $\Gamma_{0}=v \exp \left\{\left[-E_{0}+Z\left(V_{A B}+V_{B B}\right)\right] / k_{b} T\right\}$ and $\widehat{E}_{i, j}$ is an effective interaction energy defined by

$$
\widehat{E}_{i, j}=(\mathcal{M}-V) \sum_{l=1, l \in \mathcal{V}(i)}^{Z} c_{l}+(\mathcal{M}+V) \sum_{l=1, l \in \mathcal{V}(j)}^{Z} c_{l} .
$$

The internal energy of an inhomogeneous solution in a 3D atomistic model (just like SKMF) can be calculated from the interaction energies between atoms,

$$
U=\sum_{i=1}^{N} u_{i}
$$

where $N$ is the number of atoms and $u_{i}$ is the mean internal energy of site $i$. The latter can be calculated as a sum of the interaction energy of an $A$ atom on site $i$ and the interaction energy of a $B$ atom on site $i$ weighted by the finding probabilities of $A$ and $B$ on site $i$

$$
u_{i}=\frac{1}{2}\left[c_{i} E_{i}^{A}+\left(1-c_{i}\right) E_{i}^{B}\right] .
$$

Here, the factor $1 / 2$ compensates the double counting of interactions. Using expression (7) for the energies $E_{i}^{A}$ and $E_{j}^{B}$, and expression (8) for the parameters $\mathcal{M}$ and $V$, the internal energy density can be rewritten as

$$
\begin{aligned}
u_{i}= & u_{A} c_{i}+u_{B}\left(1-c_{i}\right)+c_{i}\left(1-c_{i}\right) V_{0} \\
& +\frac{1}{2}\left(\bar{c}_{l}-c_{i}\right)\left[Z \mathcal{M}+\left(1-2 c_{i}\right) V_{0}\right]
\end{aligned}
$$

where $u_{A}=Z V_{A A} / 2, u_{B}=Z V_{B B} / 2, V_{0}=Z V$, and $\bar{c}_{l}=1 / Z \sum_{l=1}^{Z} c_{l}$ is the local average concentration of $\mathrm{A}$ atoms in the vicinity of site $i$.

In a homogeneous binary solution, the probability $c_{i}$ to find an atom $A$ in site $i$ and the locally averaged probability $\bar{c}_{l}$ reduce to the average concentration $c$ of the alloy: $c_{i}=\bar{c}_{l} \equiv c$. Accordingly, the internal energy $u_{i}$ of an atom on site $i$ is equal to the density of internal energy $u$ in the homogeneous solution: $u_{i} \equiv u(c)=u_{A} c+u_{B}$ $(1-c)+c(1-c) V_{0}$. Thus, the regular solid solution model for the internal energy per atom $u(c)$ is recovered for the homogeneous solution,

$$
u(c)=u_{A} c+u_{B}(1-c)+c(1-c) V_{0} .
$$

As can be seen, the first two terms are the internal energies of the pure $A$ and $B$ components, and the excess internal energy density reads as

$$
\Delta u_{e x}(c)=c(1-c) V_{0} .
$$

Obviously, $\Delta u_{e x}(c)$ is symmetrical, so that only symmetrical solubility limits corresponding to the minima of the excess free energy can be accounted for at equilibrium. Consequently, the stationary states of the original SKMF model ${ }^{15}$ could only display symmetrical solubility limits as well.

In this article, we use a version of the original SKMF model that is modified to be applicable to asymmetric phase diagrams, ${ }^{25}$ such as that of $\mathrm{Ag}-\mathrm{Cu}$. For that purpose, we modify the expression of the MF jump frequency $\Gamma_{i, j}^{\mathrm{MF}}$, defined through Eqs. (8)-(10). This can be done by replacing the constant parameter $V_{0}$ in Eq. (15) by a composition dependent parameter $V(c)$ defined by

$$
V(c)=V_{0}+V_{1}(2 c-1)
$$

where the two coefficients $V_{0}(T)$ and $V_{1}(T)$ can be taken for example from the Redlich-Kister polynomials used in CALPHAD to reproduce the experimental solubility limits of the alloy. By doing so, the excess internal energy becomes

$$
\Delta u_{e x}(c)=c(1-c)\left[V_{0}+V_{1}(2 c-1)\right]
$$

Now, the stationary states of the SKMF model will display the same asymmetric solubility limits if taking

$$
V\left(c_{i j}\right)=V+V^{\prime}\left(2 c_{i j}-1\right),
$$

where we $V=V_{0} / Z, V^{\prime}=V_{1} / Z$ (as $V_{0}$ and $V_{1}$ defined for one atom, while $V$ and $V^{\prime}$ are defined for one bond), and

$$
c_{i j}=\frac{1}{2(Z+1)}\left[c_{i}+\sum_{l=1, l \in \mathcal{V}(i)}^{Z} c_{l}+c_{j}+\sum_{l=1, l \in \mathcal{V}(j)}^{Z} c_{l}\right]
$$

so that the interaction energy $\widehat{E}_{i, j}$ defined in Eq. (10) becomes

$$
\begin{aligned}
\widehat{E}_{i, j}= & \left(\mathcal{M}-\left[V+V^{\prime}\left(2 c_{i j}-1\right)\right]\right) \sum_{l=1, l \in \mathcal{V}(i)}^{Z} c_{l} \\
& +\left(\mathcal{M}+\left[V+V^{\prime}\left(2 c_{i j}-1\right)\right]\right) \sum_{l=1, l \in \mathcal{V}(j)}^{Z} c_{l} .
\end{aligned}
$$

In this work, the SKMF model is used to study the kinetics of phase separation in the $\mathrm{Ag}-\mathrm{Cu}$ immiscible alloy on the atomic scale. The $\mathrm{Ag}-\mathrm{Cu}$ system displays an asymmetric phase diagram, ${ }^{26}$ and the expression (20) is thus used for the interaction energy. Thermodynamic data are taken from CALPHAD to fit the experimental solubility limits of the system: ${ }^{26} f_{A g}=\tilde{f}_{A g} / N_{A}, f_{C u}=\tilde{f}_{C u} / N_{A}$, $V_{0}=\tilde{V}_{0} / N_{A}$, and $V_{1}=\tilde{V}_{1} / N_{A}$, where $N_{A}$ is Avogadro's number and $\tilde{f}_{A g}=-11945+9.67 T \mathrm{~J} / \mathrm{mol}, \tilde{f}_{C u}=-13054+9.62 T \mathrm{~J} / \mathrm{mol}$, 
$\tilde{V}_{0}=34532-9.178 T \mathrm{~J} / \mathrm{mol}$, and $\quad \tilde{V}_{1}=-5996+1.725 T \mathrm{~J} / \mathrm{mol}$. Moreover, silver and copper display very similar tracer diffusion coefficients in $\mathrm{Ag}, \mathrm{Cu}$, and the $\mathrm{Ag}$-Cu alloy. ${ }^{27,28}$ Thus, the diffusion asymmetry parameter $\mathcal{M}$ is set to 0 . Finally, the FCC lattice consists of $168^{3}$ grid points, and it is equipped with periodic boundary conditions. Equation (1) is solved in a reduced form using reduced time step $\Delta t^{*}=\Delta t \Gamma_{0}$, reduced noise amplitude $A_{n}^{*}=A_{n} / \sqrt{\Gamma_{0}}$, reduced fluxes $J_{i, j}^{*}=J_{i, j} / \Gamma_{0}$, furthermore $\mathcal{M}, V$, and $V^{\prime}$ are used in $k_{b} T$ units. The reduced time step is set to $\Delta t^{*}=0.01$, which defines the reduced time $t_{\text {SKMF }}^{*}$ of SKMF simulations, as the product between the number of iterations and the time step. Finally, when a dynamic noise is used (nucleation-growth), the reduced noise amplitude is $A_{n}^{*}=0.35$.

\section{B. Phase-field model}

In the phase-field model, the microstructure of the decomposing binary A-B system is described by the coarse grained concentration $c(\boldsymbol{r}, t)$ of component A. The temporal evolution of this concentration field is then given by the Cahn-Hilliard equation ${ }^{29}$

$$
\frac{\partial c}{\partial t}=\nabla\left(M \nabla\left[\frac{\delta F(c)}{\delta c}\right]\right) .
$$

Here, $M$ is the atomic mobility, and $F(c)$ is the total nonequilibrium free energy on the volume $\Omega$, which embodies the bulk free energy and the interfacial energy ${ }^{30}$

$$
F=\int_{\Omega}\left[f_{h}(c)+\frac{\kappa}{2}|\nabla c|^{2}\right] \mathrm{d} \Omega .
$$

The parameter $\kappa>0$ is the gradient energy coefficient and $f_{h}$ is the MFBW free energy defined by

$$
f_{h}^{(c)}=\Delta u_{e x}(c)+k_{b} T[c \ln c+(1-c) \ln (1-c)]
$$

where $\Delta u_{e x}(c)=c(1-c)\left[V_{0}+V_{1}(2 c-1)\right]$ is the internal energy emerging in the SKMF model [see Eq. (17)]. To model the kinetics of phase separation in the PFM, the phase-field equation (21) is numerically solved in its reduced form. For that purpose, the reduced free energy potential $f_{h}^{*}$ is expressed in units of $k_{b} T$,

$$
\begin{aligned}
f_{h}^{*}(c)= & c(1-c)\left[\frac{V_{0}}{k_{b} T}+\frac{V_{1}}{k_{b} T}(2 c-1)\right] \\
& +[c \ln c+(1-c) \ln (1-c)] .
\end{aligned}
$$

Besides, on introducing the characteristic time and length scales $t_{0}$ and $l_{0}$, the PFM reduced time is $t_{\mathrm{PFM}}^{*}=t / t_{0}$, and the reduced gradient operator is $\nabla^{*}=l_{0} \nabla$. Setting $t_{0}=\kappa /\left(\kappa^{*} M\left(k_{b} T\right)^{2}\right)$ and $l_{0}=\sqrt{\kappa /\left(\kappa^{*} k_{b} T\right)}$, where $\kappa^{*}$ sets the reduced width of diffuse interfaces between phases, the reduced phase-field equation is

$$
\frac{\partial c}{\partial t^{*}}=\left(\nabla^{*}\right)^{2}\left[\ln \left(\frac{c}{1-c}\right)+a_{1} c^{2}+a_{2} c-\kappa^{*}\left(\nabla^{*}\right)^{2} c\right]
$$

where $a_{1}=-6 V_{1} /\left(k_{b} T\right), a_{2}=\left(6 V_{1}-2 V_{0}\right) /\left(k_{b} T\right)$ from the first derivative of $f_{h}^{*}(c)$ in Eq. (24). Here, the mobility is assumed constant, as it was justified for the Ag-Cu system in Ref. 27.

The PFM model is used to study the kinetics of phase separation in the Ag-Cu immiscible alloy at the microstructure scale. In Eq. (21), $c(\boldsymbol{r}, t)$ is the local concentration of silver. All thermodynamic parameters are thus the same as in the SKMF model. Moreover, the mobility $M$ for this system was computed as a function of temperature $T$ in Ref. 27. As for the parameter $\kappa$, it will be computed through the multiscale procedure in what follows. Phase-field simulations are performed by numerically solving the nonlinear equation (25) for $\kappa^{*}=1.0$, using the Spectral-Eyre (SE) scheme. $^{31}$ The $3 \mathrm{D}$ simulation box consists of $168^{3}$ grid points. It is equipped with periodic boundary conditions. A uniform reduced time step $\Delta t^{*}=0.02$, which defines the reduced time $t_{\mathrm{PFM}}^{*}$ of PFM simulations, and a mesh size $\Delta x^{*}=\Delta y^{*}=\Delta z^{*}=0.97$ are chosen. This choice of space step will allow to match the size of the PFM simulation box with that of SKMF in real units (see the paragraph on the length scale of the multiscale method). In the spinodal region, the initial state is a homogeneous solution with small composition fluctuations of amplitude 0.002 , centered in the average composition $\bar{c}$ of silver.

\section{Sequential MS procedure}

The multiscale (MS) procedure proposed in this work is sequential: numerical methods (SKMF and PFM) operate at their natural scale (atomistic and mesoscopic, respectively) and are connected via the upstream transfer of relevant parameters.

The procedure first consists of connecting the length and time scales of SKMF and PFM. Second, it proceeds by simulating the first stages of the decomposition dynamics on the atomic scale by means of the SKMF simulations, and then using this simulation output as the initial solution of PFM simulations for the latest stages of the dynamics. Third, the MS procedure is upscaled, by using the particle size distribution (PSD) and density as an input for PFM simulations on a larger simulation box.

The first step of the MS procedure guarantees the consistent integration of the two models within the global approach. Besides, it provides the MS procedure with its space and time scales in real units. A choice was made to connect the SKMF and PFM on the elementary case study of the growth of a single spherical germ. In details, the SKMF atomistic model intrinsically contains its own length scale in real units, through the $3 \mathrm{D}$-lattice. On the contrary, the resolution of the PFM is the spatial correlation length $\left(\simeq l_{0}\right)$. This characteristic length scale is thus chosen to be the pivot between the two methods. It is connected to the natural length scale of the SKMF model, by matching the interface profiles between the copper-rich precipitate and the silver-rich matrix as simulated by both methods. Next, the time scales of SKMF and PFM are connected by matching the time evolution of the precipitate radius in both cases. An estimation of the interatomic diffusion coefficient for the $\mathrm{Ag}-\mathrm{Cu}$ alloy, calculated in a former work $^{27}$ is also used to express the time scale of the MS procedure in real units. Moreover, the equivalence between the stationary states of the SKMF on the atomic scale and the absolute minimum of the Mean-Field Bragg-Williams (MFBW) free energy 
used in the PFM ensure the thermodynamical consistency of the MS procedure.

The second level of the MS procedure is to feed the PFM with the atomistic simulations of the SKMF. In that case, SKMF and PFM simulations are performed on simulation boxes with the same size $\left(168^{3}\right.$ grid points in this work) and connected at the instant corresponding to the maximum number of particles. This strategy is nonetheless limited by the computational load of SKMF simulations on a big simulation box. To upscale the MS procedure, the PSD and number of precipitates provided by SKMF at the maximum number of particles is then used as the input for the PFM simulations on a bigger simulation box $\left(512^{3}\right.$ in this work).

\section{PARAMETRIZATION OF THE MS PROCEDURE FOR Ag-Cu}

\section{A. Solubility limits and nucleation-growth domain}

The solubility limits in the Ag-Cu alloy were computed using both SKMF and PFM independently, by taking the maximum and minimum values of the bulk concentration. In both cases, the criterion for reaching the equilibrium concentration in the bulk of the precipitates and the matrix was the stationarity of the numerical solubility limits. This criterion was met after roughly 30000 iterations by the SKMF model and 5000 iterations by the PFM. Results for the SKMF and the PFM are displayed in Fig. 1 using blue squares and red circles, respectively (full marks), vs the experimental binodal curve taken from Ref. 26 (continuous line).

In the temperature range of solid $\mathrm{Ag}-\mathrm{Cu}(T<1050 \mathrm{~K})$, the solubility limits of both SKMF and PFM are in good agreement with the experimental phase diagram. This is not surprising, considering that the MFBW free energy density that determines the equilibrium state of the PFM, as well as the equivalent stationary states of the SKMF model, were parametrized using the same data

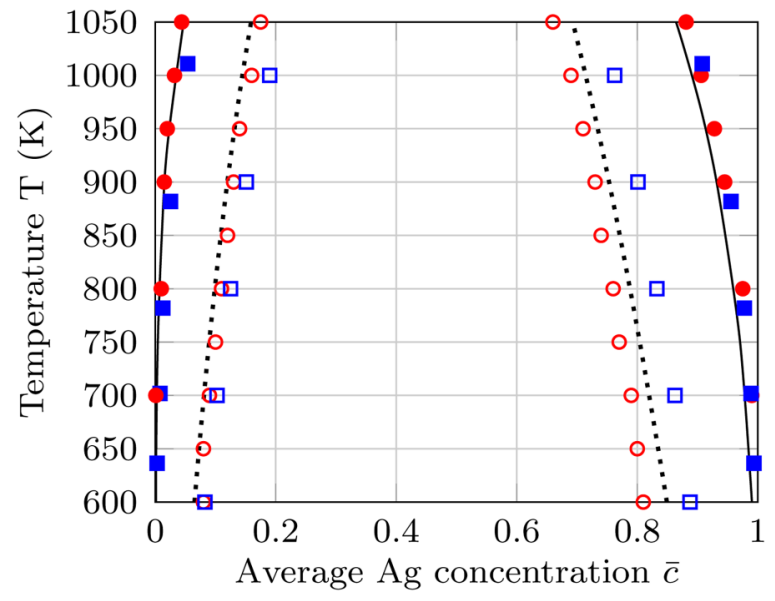

FIG. 1. Solubility limits (full marks) and spinodal limit (empty marks) for different temperatures in the Ag-Cu system, as provided by PFM (red circles) and SKMF simulations (blue squares), vs the binodal line provided by CALPHAD (black line), and the spinodal line provided by the $\mathrm{CH}$ linear theory (black dots). taken from CALPHAD. In particular, the asymmetry of the $\mathrm{Ag}-\mathrm{Cu}$ phase diagram is accounted for.

The spinodal limit was also determined using SKMF and PFM. For that purpose, simulations were performed using different average concentrations and at different temperatures. In the PFM, the initial condition was a homogeneous solution with small fluctuations of composition of amplitude 0.002 (static noise). In SKMF, a homogeneous system with a $10^{-6}$ fluctuation on a single site was used without dynamic noise $\left(A_{n}=0\right)$. Only concentration-temperature couples allowing phase separation were considered to be inside the spinodal region. Incidentally, this procedure allowed to circumscribe the nucleation-growth domain of the Ag-Cu system.

It should be mentioned that, when approaching the binodal line from the metastable region using SKMF without dynamic noise, the system might be stuck in a metastable state, thereby blurring the delimitation between metastable and unstable regions in the phase diagram. To evaluate this bias, the same study of the binodal line was also performed using SKMF with dynamic noise (not presented). Results with and without dynamic noise proved very similar, and this bias could then be neglected. The simulations gave somewhat different compositions than the linear $\mathrm{CH}$ theory (dotted line) for both methods. The discussion of this disparity is beyond the scope of the present paper and some aspects of it is already discussed elsewhere. ${ }^{25}$ In the following, simulations are performed at $T=873 \mathrm{~K}$, and for an average concentration $\bar{c}=0.83$, which places the system outside of the spinodal limit in the nucleation-growth domain for both methods.

\section{B. Space scale}

In the SKMF model, the length scale is naturally set by the lattice spacing. In the case of the Ag-Cu alloy, the average lattice parameter of the FCC lattice is about $a_{0}=3.8 \AA^{32}$ so that $\Delta x=a_{0} / 2=0.19 \mathrm{~nm}$. Consequently, the lattice grid used for the SKMF approach $\left(168^{3}\right.$ sites) roughly corresponds to a $(32 \mathrm{~nm})^{3}$ spatial domain. In the PFM, the space scale is determined by the upstream setting of the correlation length by means of an atomistic approach, chosen to be the SKMF model here. Note that in reality, during the decomposition, the lattice constant changes as its value in pure $\mathrm{Cu}$ is $3.597 \AA$ and it is $4.079 \AA$ in pure $\mathrm{Ag} .{ }^{33}$ In our simulations, the lattice misfit was neglected, and no elastic effects were considered.

For that purpose, the growth of a single precipitate of copper is simulated using SKMF, for an average concentration $\bar{c}=0.83$ and at $T=873 \mathrm{~K}$. The resulting equilibrium profile of the $\mathrm{Ag} / \mathrm{Cu}$ interface in the $\langle 100\rangle$ direction is materialized by blue squares in Fig. 2. The criterion for reaching the equilibrium interface was the stationarity of the interface width and solubility limits. At this temperature, and for $\bar{c}=0.83$, this requirement was met after roughly 30000 iterations. The SKMF interface profile was then fitted by a typical hyperbolic tangent function reproducing the PFM interface profile. In details, the interface ansatz was $I(x)=A_{0}+A_{1} \tanh \left(x / l_{0}\right)$ (correlation factor $\rho>0.999$ ), where the characteristic length scale was found to be $l_{0}=0.196 \mathrm{~nm}$ (red line in 2). The choice to take $\Delta t^{*}=0.97$ thus provides $\Delta x_{\mathrm{PFM}}=\Delta x_{\mathrm{SKMF}}=1.90 \times 10^{-10} \mathrm{~m}$ (see Table I). Though singular, this small space step for the PFM is the price to pay for fitting the composition values of SKMF on the 


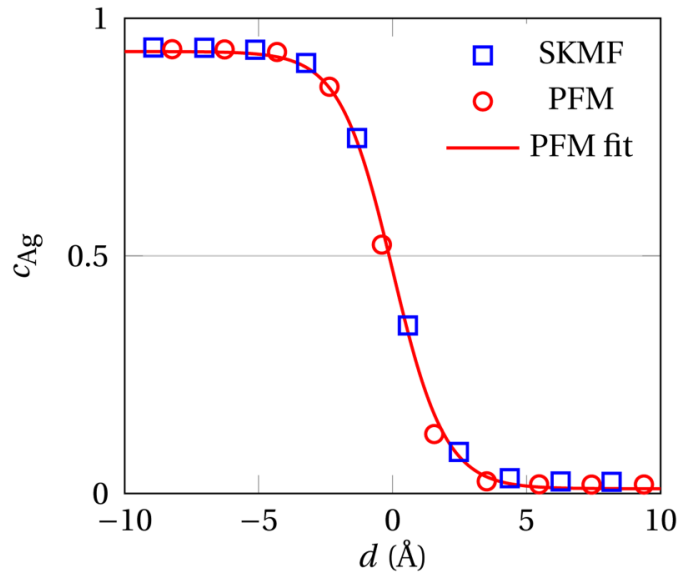

FIG. 2. Ag/Cu interface equilibrium profile as obtained by SKMF simulations (blue squares) for $\bar{c}=0.83$, at $T=873 \mathrm{~K}$, fitting (correlation factor $\rho>0.999$ ) by the PFM tanh profile (red line) and the resulting PFM profile (red circles).

atomic scale via the continuous and derivable functions solving the phase-field equation (21). In passing, a similar value was used in previous PFM based MS procedures. ${ }^{6}$, However, in no case does it attest for the applicability of the PFM on the atomic scale.

To justify the transfer of the composition profile from the atomistic SKMF to the continuum PFM, we must run SKMF until it reaches a state where the interface is smooth enough to serve as an adequate initial condition for the numerical solution of PFM. It was shown before that continuum equations are valid on the atomic scale if diffusion distances are longer than a few times the atomic distance. ${ }^{34,35}$ We must note, however, that this distance might depend strongly on the diffusion asymmetry (composition dependence of the diffusion coefficient). ${ }^{36,37}$ As in the Ag-Cu system, the diffusion asymmetry is negligible, that is $\mathcal{M}=0$, the former case holds. We have chosen the runtime of SKMF simulations accordingly. This is notably supported by the diffuse interface profile that naturally arises in atomistic SKMF simulations where Fick's continuous diffusion is not assumed and which matches the hyperbolic tangent profile of phase field.

In these conditions, the $168^{3}$ simulation box used in both PFM and SKMF simulations gives roughly a $(32 \mathrm{~nm})^{3}$ spatial domain. The corresponding PFM interface is also displayed (red circles) in Fig. 2.

The gradient energy coefficient $\kappa$ can be derived from $l_{0}$, using that $\kappa=k_{b} T l_{0}^{2}$, which leads to $\kappa=4.6 \times 10^{-21} \mathrm{~J} \mathrm{~nm}^{2}$ or $\kappa=2.1 \mathrm{eV} \mathrm{nm}^{-1}$ at $T=873 \mathrm{~K}$ (density is $72.9 \mathrm{~nm}^{-3}$ for $\mathrm{Ag}-\mathrm{Cu}$ ). This is slightly higher than $\kappa=1.5 \mathrm{eV} \mathrm{nm}^{-1}$ as obtained via

TABLE I. Space and time steps for the SKMF and PFM, at $T=873 \mathrm{~K}$.

\begin{tabular}{cc}
\hline \hline SKMF & PFM \\
\hline$\Delta t=9.03 \times 10^{-6} \mathrm{~s}$ & $\Delta t=6.32 \times 10^{-5} \mathrm{~s}$ \\
$\Delta x=1.90 \times 10^{-10} \mathrm{~m}$ & $\Delta x=1.90 \times 10^{-10} \mathrm{~m}$ \\
\hline \hline
\end{tabular}

MC/MD calculations in Ref. 27 at $T=900 \mathrm{~K}$. It results from stiffer interfaces in the SKMF model compared to the procedure used in Ref. 27. In passing, it was observed in Ref. 27 that for temperatures ranging from 600 to $1000 \mathrm{~K}, \kappa$ could be considered independent of the temperature. We thus suggest that this constant value of $\kappa$ can be used in this range of temperature to compute back the length scale $l_{0}$ for any temperature.

\section{Time scale}

To provide the time scale $t_{0}$ of PFM simulations in real units for the $\mathrm{Ag}-\mathrm{Cu}$ alloy, the atomic mobility $M$ is estimated in the standard framework of Martin ${ }^{18}$ for bulk diffusion in a binary alloy,

$$
M \simeq \frac{\bar{c}(1-\bar{c})}{k_{b} T} \bar{D}
$$

Here, $\bar{D}$ is the chemical interdiffusion coefficient. It is assumed to be independent of the local concentration of silver and copper in the vicinity of the interface. This is justified by similar tracer diffusion coefficients for copper and silver in copper, silver, and silver-copper. This approximation is consistent with the choice to set the diffusion anisotropy parameter $\mathcal{M}$ to zero in the SKMF model [see Eq. (10)]. Under this approximation, the following estimation of the chemical interdiffusion coefficient was derived in Ref. 27: $\bar{D}(T=873 \mathrm{~K})=75.9 \mathrm{~nm}^{2} \mathrm{~s}^{-1}$. The expression (26) then gives the atomic mobility at $T=873 \mathrm{~K}: M=161 \mathrm{~nm}^{2} \mathrm{~s}^{-1} \mathrm{eV}^{-1}$, and the time scale of the PFM procedure follows $t_{0}=l_{0}^{2} /\left(k_{b} T M\right)=3.16 \times 10^{-3} \mathrm{~s}$. By definition, $t_{0}$ also sets the time scale of the MS procedure.

The consistent integration of the SKMF model within the MS procedure requires that its time scales is connected to the time scale of the PFM. To do so, the growth of a single precipitate is simulated using SKMF and PFM, at $T=873 \mathrm{~K}$, and for an average concentration $\bar{c}=0.83$. To estimate the precipitate radius, the limit between the precipitate and the matrix is set at half interface. The same initial spherical nucleus of radius $R(t=0)=3.8 \mathrm{~nm}$ (20 grid spacing) by far superior to the critical radius for nucleation, and bulk concentration 0.013 given by the lower solubility limit of the alloy is used in both SKMF and PFM. This initial condition corresponds to the equilibrium shape and bulk concentration of the precipitate. It ensures that only the growth process by means of Fickian diffusion is modeled, except for the first iterations when the interface of the nucleus aligns on its equilibrium profile (Fig. 2). Also, the noise amplitude is set to zero in the SKMF simulations for consistency with the PFM.

The time evolution of the precipitate radius $R(t)$ as simulated by SKMF and PFM is displayed in Fig. 3. The two curves match, provided that the reduced time scale in the SKMF simulations was preliminary rescaled by a factor $\tau_{0}=3.50 \pm 0.01$. This proves that the reduced time scales of SKMF and PFM are in a proportionality relationship $t_{\mathrm{SKMF}}^{*}=\tau_{0} t_{\mathrm{PFM}}^{*}$. After that, the SKMF model could, in turn, be equipped with the real time scale $t_{0}$ of the PFM, as derived from the atomic mobility $M$, resulting in Fig. 3. Alternatively, the real time scale of the SKMF approach could have been estimated independently, by explicitly calculating the MF jump frequency $\Gamma_{i, j}^{\mathrm{MF}}$ [see formula (9)] and then compared to the PFM time scale $t_{0}$. However, in this work, a choice was made to "impose" the same 


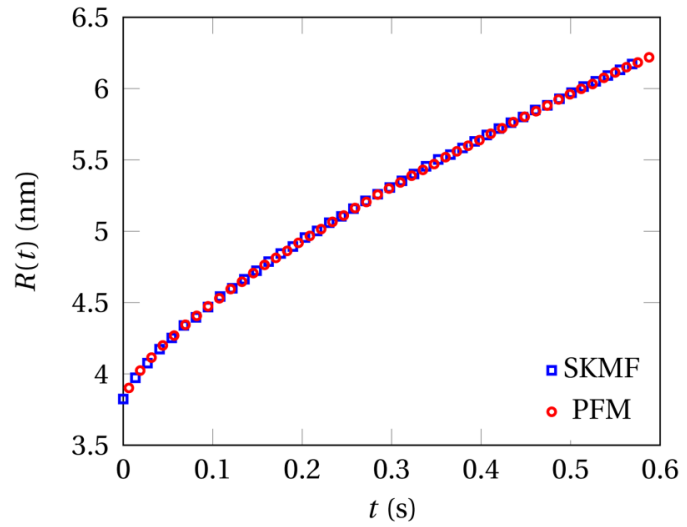

FIG. 3. Time evolution of the radius $R(t)$ of a single growing precipitate, for $\bar{c}=0.83$, at $T=873 \mathrm{~K}$, as provided by SKMF simulations (blue squares) after rescaling by a factor $\tau_{0}=3.50$ and PFM simulations (red circles).

time scale to both methods, thereby ensuring the consistent connection between the simulation of the early and later stages of the system kinetics, by means of the SKMF and PFM, respectively.

Considering that $\Delta t_{\mathrm{SKMF}}^{*}=0.01$ and $\Delta t_{\mathrm{PFM}}^{*}=0.02$, the shared time scale for SKMF and PFM in real units can be displayed in Table I through their respective time steps. In particular, it can be seen that the time step for the PFM is roughly seven times as big as that of SKMF: $\Delta t_{\mathrm{PFM}}=7.00 \times \Delta t_{\mathrm{SKMF}}$. SKMF simulations were also performed using a dynamic Langevin noise for the single precipitate growth, and the same time scale as in the previous case was obtained. However, due to the stochastic nature of SKMF, additional nuclei appeared. In this case, the time scale was fitted for the growth of the first precipitate. It should also be noted that the two curves for $R(t)$ in Fig. 3 align on a square root law of time.
This validates the hypothesis of the Fickian diffusion regime, as predicted in the Wagner theory for the growth of a single precipitate. $^{38}$

\section{MS SIMULATION OF NUCLEATION-GROWTH IN $\mathrm{Ag}-\mathrm{Cu}$}

\section{A. SKMF simulations}

SKMF simulations have been performed at $873 \mathrm{~K}$ annealing temperature. A total of $8.75 \cdot 10^{6}$ iterations were necessary to investigate the nucleation of $\mathrm{Cu}$ rich clusters in the Ag matrix. As one can see it in Fig. 1, the initial $\bar{c}=0.83$ average composition is inside the miscibility gap but outside of the spinodal region for both methods. In order to overcome the nucleation barrier, dynamic noise was used. The nucleation time depends on both the noise amplitude and the chemical driving force. The latter is higher for $(\bar{c}, T)$ couples close to the binodal curve in the phase diagram. At a given temperature, a higher $\bar{c}$ leads to a longer nucleation time, whereas a higher $A_{n}$ usually means faster formation of viable $\mathrm{Cu}$ rich precipitates but worse statistics. Figure 4(a) shows the appearance of the first small nuclei $(<1 \mathrm{~nm})$.

Then, additional nuclei keep forming while the latter grow rapidly, resulting in an increasing mean radius $\tilde{R}$ [Fig. 5(a)], until the simulation reaches the maximum number of $\mathrm{Cu}$ rich precipitates [Fig. 5(b)]. This step ( $t=0.16 \mathrm{~s})$ was taken as the initial state of PFM simulations (see Sec. IV B). Contrary to the isolated growing precipitate, SKMF simulation continued with $A_{n} \neq 0$, while no dynamic noise was used in PFM. Thereafter, the number of precipitates decreases, while $\tilde{R}$ increases at the same time, corresponding to the coarsening process.

Figure 4(a) shows that at the early stages $(<0.05 \mathrm{~s})$, no nucleus can be observed. After the appearance of the first nucleus, the system quickly $(\sim 0.1 \mathrm{~s})$ reaches the maximum number of precipitates, where the average size of the particles is around $1.5 \mathrm{~nm}$.

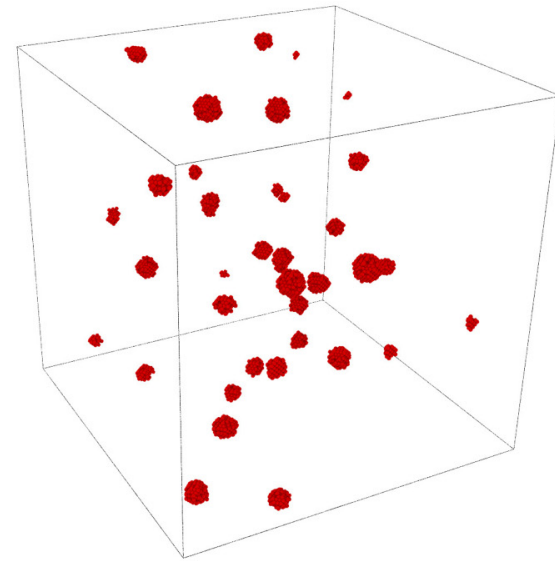

(a)

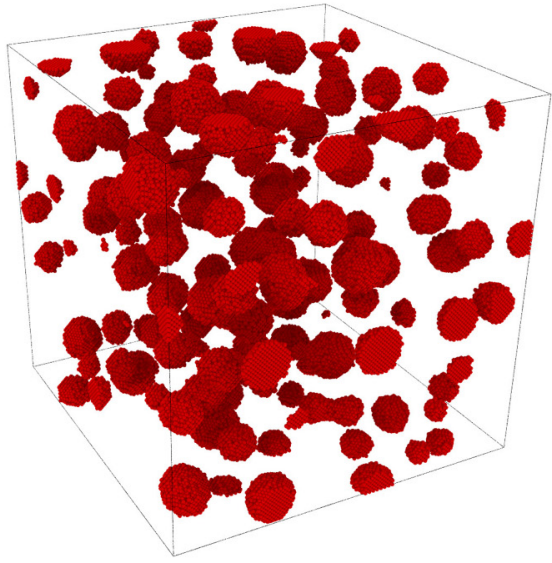

(b)

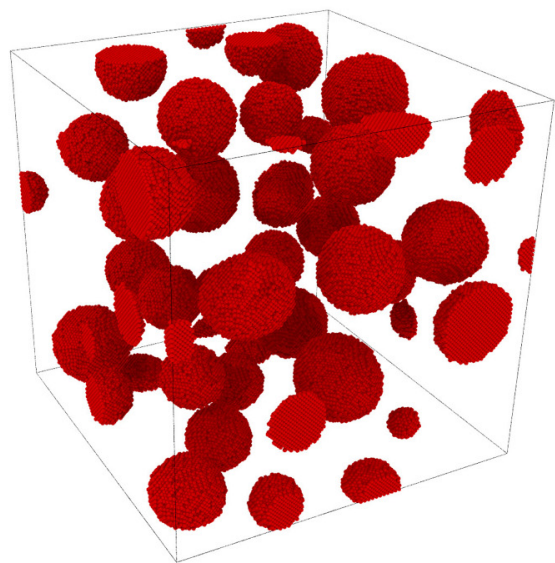

(c)

FIG. 4. Microstructures obtained by SKMF simulations using a dynamic noise of amplitude $A_{n}^{*}=0.35$, at $T=873 \mathrm{~K}$, for $\bar{c}=0.83$, at times (a) $t=0.05 \mathrm{~s}$, (b) $t=0.16 \mathrm{~s}$, and (c) $t=7.90 \mathrm{~s}$. 


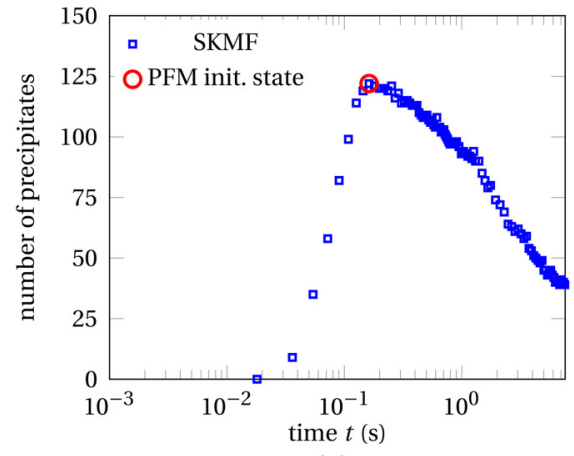

(a)

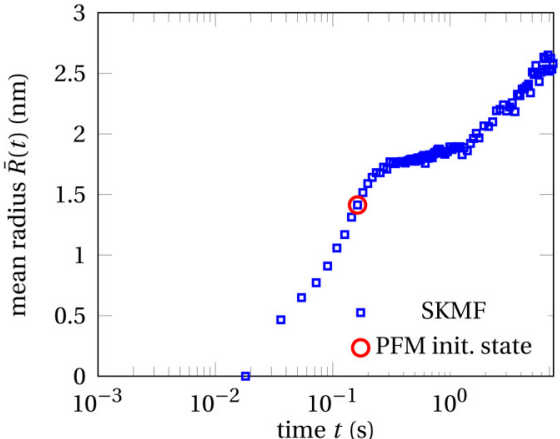

(b)

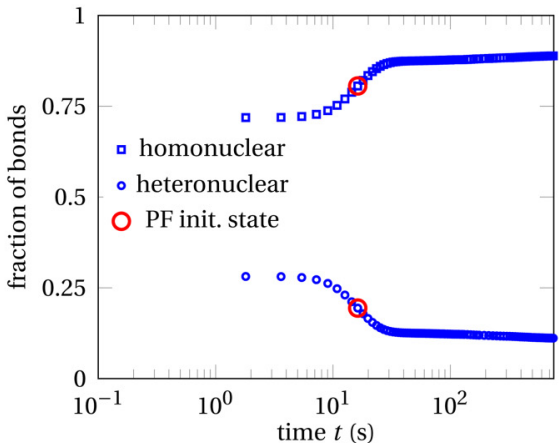

(c)

FIG. 5. (a) Number of precipitates, (b) mean precipitate radius, (c) fraction of homo and heteronuclear bonds, as obtained by SKMF simulations (blue squares) using a dynamic noise of amplitude $A_{n}^{*}=0.35$, for $\bar{c}=0.83$, at $T=873 \mathrm{~K}$. Red circle: initial configuration for PFM simulations.

This is followed by a slow coarsening process, while the mean radius of the precipitates increases [Fig. 4(b)]. The fraction of AA, $\mathrm{BB}$, and $\mathrm{AB}$ bonds can be calculated for the whole system based on the probabilistic approach of SKMF. Between sites $i$ and $j$, the value of $c_{i} c_{j},\left(1-c_{i}\right)\left(1-c_{j}\right)$, and $c_{i}\left(1-c_{j}\right)+\left(1-c_{i}\right) c_{j}$ gives the fraction of $\mathrm{Ag}-\mathrm{Ag}, \mathrm{Cu}-\mathrm{Cu}$, and $\mathrm{Ag}-\mathrm{Cu}$ bonds, respectively. The formation of homonuclear $(\mathrm{Ag}-\mathrm{Ag}$ and $\mathrm{Cu}-\mathrm{Cu})$ bonds is energetically advantageous, thus the system wants to minimize the number of unfavorable heteronuclear $\mathrm{Ag}-\mathrm{Cu}$ bonds, as it is presented in Fig. 5(c).

\section{B. PFM simulations}

Consistently with SKMF, PFM simulations were performed using $\bar{c}=0.83$ and $T=873 \mathrm{~K}$. The initial conditions was the SKMF microstructure at $t=0.16 \mathrm{~s}$ [Fig. 4(b)]. In order to transfer the solution from SKMF to PFM, we used the fact that in the SKMF approach, the atom occupation probability varies smoothly from one site of the FCC lattice to another. It was thus possible to interpolate the atomic coordinates on the empty lattice sites into a quasicontinuous configuration for subsequent PFM simulations [Fig. 6(a)]. One should keep in mind that this procedure to transfer the solution from SKMF to PFM relies upon the same justification we used in Sec. III B to connect the space scales of both methods by matching the interface profile of a single growing precipitate.

Moreover, the PFM coarse grained concentration $c$ is a continuous field defined at the mesoscopic level, which is discretized on a simple cubic lattice afterwards. Thus, the connection of the SKMF and PFM not only requires to interpolate the SKMF concentration from the FCC lattice to the simple cubic lattice, but it also virtually changes the nature of the variable describing the system. This discussion is not trivial, but it is beyond the scope of this work. The time $t=0.16 \mathrm{~s}$ corresponds to the maximum number of precipitates in the SKMF simulation (122). The evolution of the microstructure simulated by the PFM is displayed in Fig. 6.

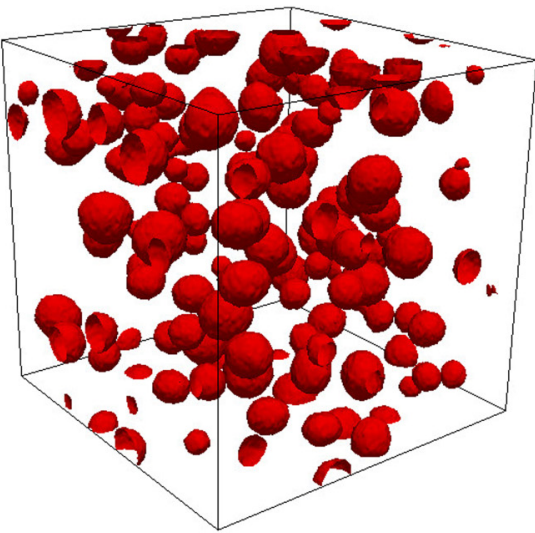

(a)

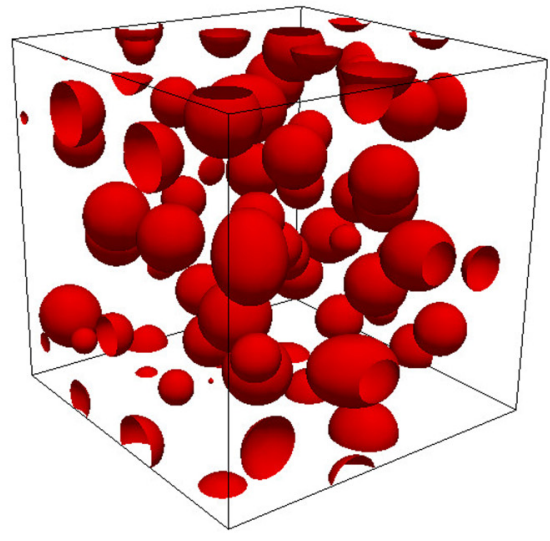

(b)

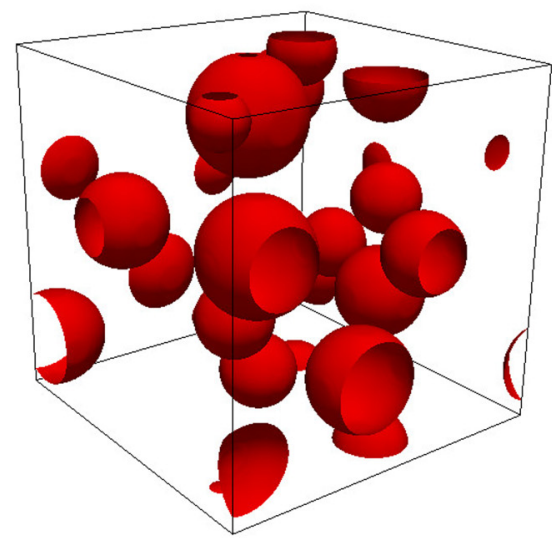

(c)

FIG. 6. Microstructures obtained by PFM simulations using as initial condition the microstructure as obtained by SKMF at $t=0.16 \mathrm{~s}$, at $T=873 \mathrm{~K}$, for $\bar{c}=0.83$, at times (a) $t=0.16 \mathrm{~s}$, (b) $t=2.69 \mathrm{~s}$ and (c) $t=19.15 \mathrm{~s}$. 
Between $t=0.16 \mathrm{~s}$ and $t \simeq 2 \mathrm{~s}$, the system undergoes a short transient regime. It is characterized by the decrease of the volume fraction of precipitates and numerous denucleation events for small particles. Then, the coarsening regime is initiated, where the volume fraction of precipitates remains steady, bigger particles grow, and smaller particles denucleate or coalesce.

To quantify this trend, the PSD of the system is shown in Fig. 7(a) at different times. The initial distribution for PFM simulations is given in the inset. It can be seen that the distribution flattens and migrates toward bigger particles with time. This process is usually described by the Lifshitz Slyozov Wagner (LSW) theory, ${ }^{39}$ stating that the microstructure is self-similar during the coarsening stage. As a corollary, the reduced PSD scaled by the

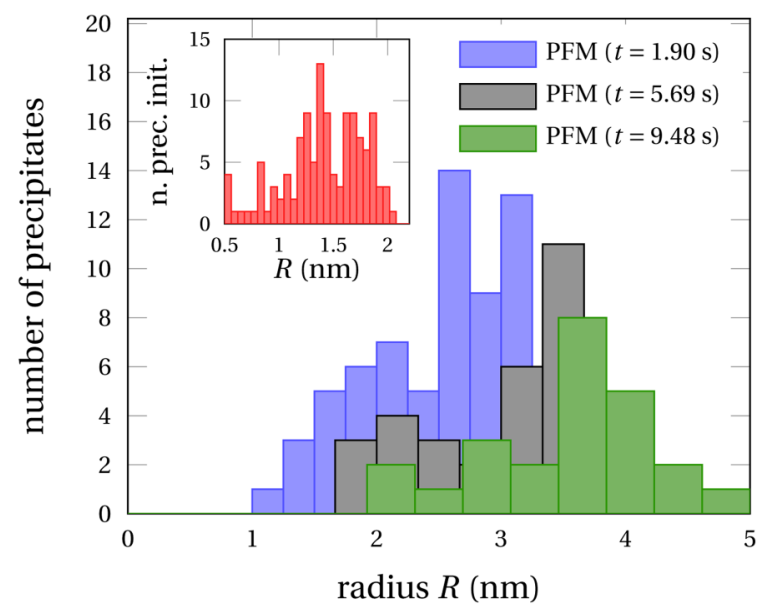

(a)

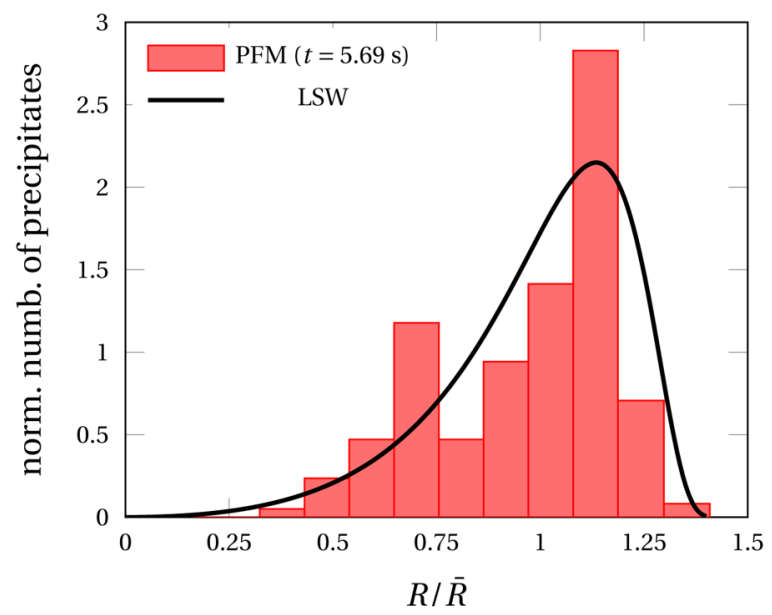

(b)

FIG. 7. Particle size distribution in PFM simulations, at $T=873 \mathrm{~K}$, for $\bar{c}=0.83$, (a) at different times (inset: initial particle size distribution in PFM as taken from SKMF simulations), and (b) scaled by the mean radius, vs the theoretical particle size distribution in the LSW theory (black line). mean radius $\bar{R}$ (red histogram) follows the LSW theoretical distribution (black line) in Fig. 7(b).

The variation of the number of precipitates and mean precipitate radius $\bar{R}$ is also plotted in Fig. 8. During the first transient regime, the number of precipitates plummets, while the mean radius raises fast to $2.1 \mathrm{~nm}$. Then, the growth dynamics slows down during the coarsening regime. In Fig. 8(b), the formation of steps corresponds to the concomitant coalescence of a cluster of precipitates, when the statistics becomes insufficient. The evolution of the mean radius $\bar{R}$ as simulated by PFM (red circles) is also compared to the LSW theoretical law $\bar{R}(t)=\left(\bar{R}_{c}^{3}+K t\right)^{1 / 3}$ (black line), where $\bar{R}_{c}=2.27 \mathrm{~nm}$ is the mean radius at the beginning of the coarsening stage and $K=2.78 \mathrm{~nm}^{3} \mathrm{~s}^{-1}$ is the LSW rate constant. The fit procedure was performed between $t=1 \mathrm{~s}$ and $t=10 \mathrm{~s}(\rho>0.99)$. PFM results are in good qualitative agreement with the LSW theory.

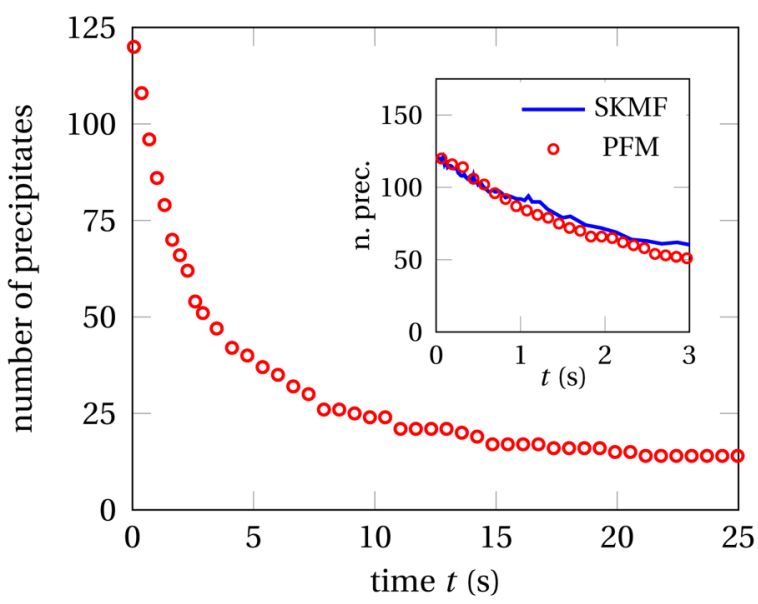

(a)

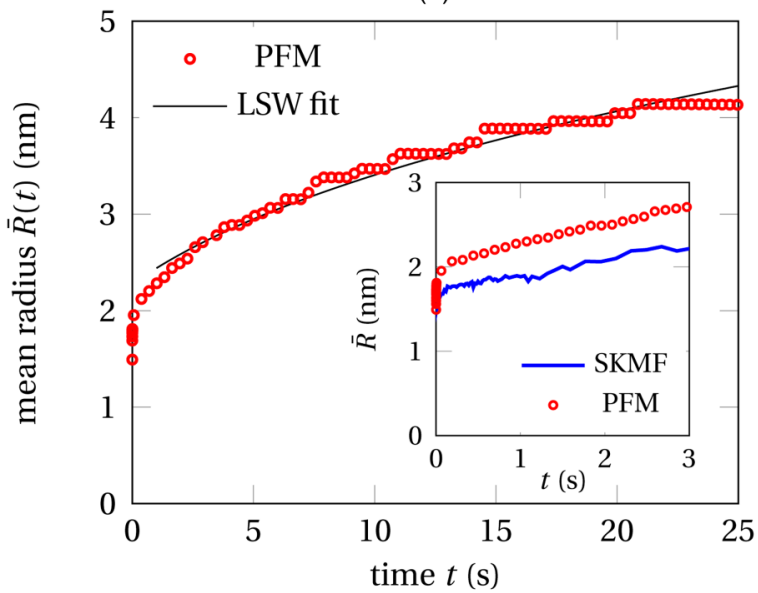

(b)

FIG. 8. (a) Number of precipitates and (b) mean precipitate radius, as obtained by PFM (red circles) for $\bar{c}=0.83$, at $T=873 \mathrm{~K}$. Black line: LSW fit. Inset: comparison between PFM (red circles) and SKMF (blue line) simulations at early stages of dynamics. 


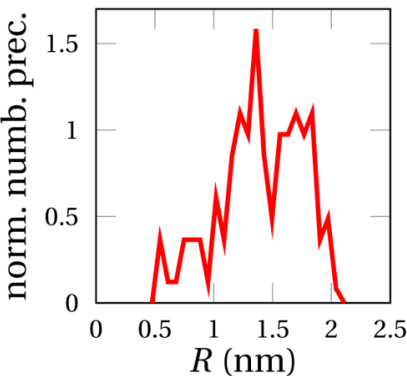

(a)

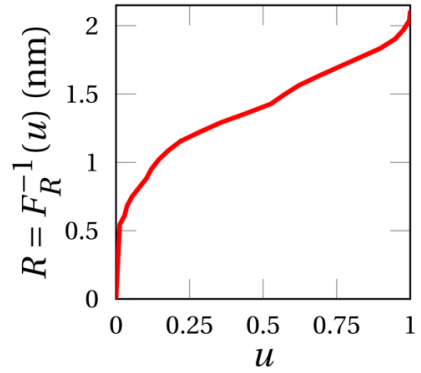

(b)
FIG. 9. (a) Initial PSD for PFM upscaled simulations and (b) corresponding inverse partition function $F_{R}^{-1}(u)$ used on a random trial $u$ with uniform law.

It should be noted that the rate constant $K$ is high here. This is due to $\bar{c}=0.83$ being close to the spinodal limit at $T=873 \mathrm{~K}$, as a high volume fraction of the second phase (copper here) was shown analytically ${ }^{40-42}$ and numerically ${ }^{6,43,44}$ to increase the rate constant, sometimes up to one order of magnitude. Moreover, the analytical models in Ref. 41 also predict a more symmetric and broader normalized PSD than that expected from the original LSW. This is consistent with the present work [see Fig. 7(b)].

To allege for the self-consistency of the MS procedure, SKMF simulations were extended to the early stages of the coarsening process (insets in Fig. 8). It can be seen that the total number of precipitates as provided by PFM (red circles) and SKMF (blue line) are in good agreement, although in SKMF these values are somewhat higher. The mean radius only differs by a roughly constant gap. These disparities are linked with the presence of noise in the SKMF simulations, which maintains the nucleation process during the coarsening regime. A stricter comparison between the SKMF and PFM simulations of the coarsening stage would have required to remove the dynamic noise from the SKMF simulations after $t=0.16 \mathrm{~s}$ corresponding to the connecting time between SKMF and PFM, as the silver matrix subsequently reaches thermal equilibrium, so that almost no further nucleation is expected and Ostwald ripening can be considered as the dominant precipitation process. The observed compatibility between SKMF and PFM results nonetheless tend to support the self-consistency of the MS procedure.

\section{Upscaling the MS procedure}

The second step of the MS procedure is to integrate the PSD and precipitate density as provided by SKMF in PFM simulations. Indeed, the stumbling block of the MS procedure developed hitherto is the computational cost of SKMF simulations when a large simulation box is used. To circumvent this shortcoming, only the PSD and the precipitate density taken from SKMF are upscaled from atomic to mesoscopic level. This original approach allows to use an arbitrarily bigger simulation box for PFM calculations. Consistent with the integrated MS procedure, the SKMF microstructure at $t=0.16 \mathrm{~s}$ was considered here. It contains 122 particles in a volume of $32^{3}=32768 \mathrm{~nm}^{3}$, corresponding to a precipitate density of $3.72 \times 10^{-3}$ prec. $\mathrm{nm}^{-3}$. Here, PFM simulations were performed on a $512^{3}$ computational grid, corresponding to a volume of roughly $100^{3} \mathrm{~nm}^{3}$. Using the same precipitate density, the PFM simulation box initially contained 3723 particles, thereby ensuring a good statistics. The associated PSD is shown again in Fig. 9(a). For the initial state of PFM simulations, 3723 particles were generated and distributed uniformly in the simulation box. Moreover, the radius of each particle was chosen randomly, following the PSD of SKMF.

For that purpose, the inverse $F_{R}^{-1}$ of the cumulative distribution function $F_{R}(r)=\mathbb{P}(R \leq r)$ ( $\mathbb{P}$ being the probability) associated to the PSD of SKMF was determined [see Fig. 9(b)]. Then, each particle radius $R$ was drawn as $R=F_{R}^{-1}(u)$, where $u$ is a random trial of the uniform law between 0 and 1 . The evolution of

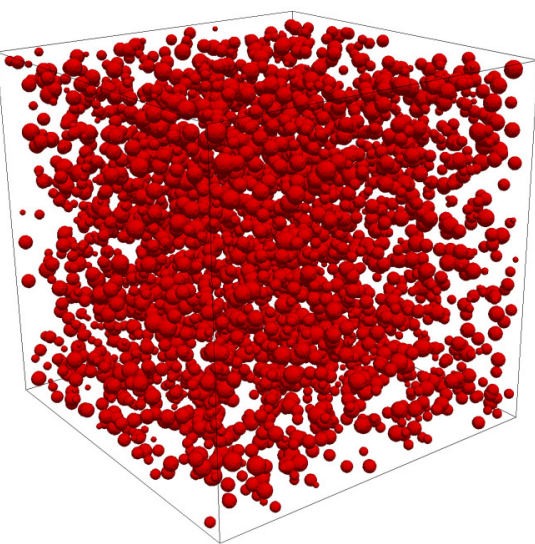

(a)

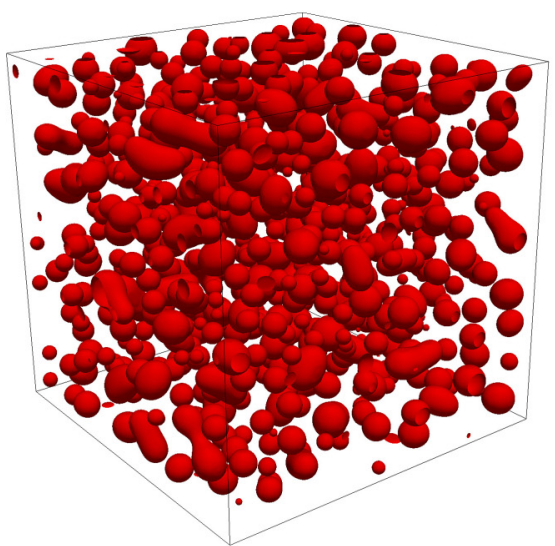

(b)

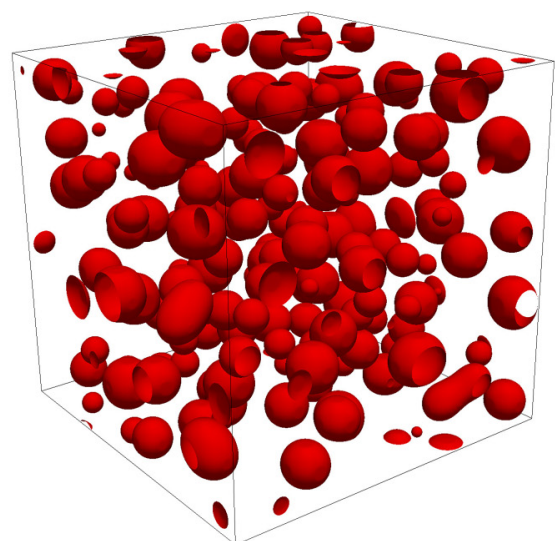

(c)

FIG. 10. Microstructures obtained by PFM simulations on a $512^{3}$ grid point simulation box using as initial condition the number of precipitates and PSD as obtained by SKMF on a $168^{3}$ grid point simulation box, at $T=873 \mathrm{~K}$, for $\bar{c}=0.83$, at times (a) $t=0.16 \mathrm{~s}$, (b) $t=1.26 \mathrm{~s}$, and (c) $t=17.7 \mathrm{~s}$. 
the microstructure simulated by the PFM on a $100^{3} \mathrm{~nm}^{3}$ computation grid is displayed in Fig. 10, at time $t=0.16 \mathrm{~s}$ (PFM initial state), $t=3.37 \mathrm{~s}$, and $t=19.15 \mathrm{~s}$.

\section{CONCLUSION}

In this work, we reported a quantitative multiscale procedure based on the recently developed SKMF approach, combined with the PFM, and parametrized via CALPHAD database, to simulate the nucleation-growth-coarsening process in alloys. The SKMF was chosen as an alternative to other atomistic approaches such as $\mathrm{KMC}$ to reproduce the nucleation stage of the kinetics, as it can be integrated more easily within a PFM based MS approach. Moreover, the PFM was used at the mesoscopic level to simulate the subsequent coarsening process, as it can address bigger space and time scales. The originality of the proposed MS procedure is threefold. First, the space and time scales were explicitly matched between atomic and mesoscopic levels. Second, the thermodynamic consistency was guaranteed by adjusting on the same thermodynamic data taken from CALPHAD, both the effective interaction energy in the SKMF model and the free energy in the PFM. Third, the postnucleation microstructure simulated by SKMF was explicitly transferred to the PFM. Alternatively, only the PSD and the precipitate density taken from SKMF were fed into PFM simulations, thereby allowing to use bigger simulation boxes for PFM calculations. The developed procedure was applied to the $\mathrm{Ag}-\mathrm{Cu}$ model alloy, for which a ready-to-use version was proposed in this article. In this case, the coarsening process at high volume fractions of precipitates for which analytical results become scarcer could be simulated.

\section{ACKNOWLEDGMENTS}

The authors are grateful for the support of the TÉT_16-12016-0100 French-Hungarian TéT_16_FR/Balaton project. The research was financed by the Higher Education Institutional Excellence Program of the Ministry of Human Capacities in Hungary, within the framework of the Energetics thematic program of the University of Debrecen.

\section{REFERENCES}

${ }^{1}$ G. Lu and E. Kaxiras, "An overview of multiscale simulations of materials," (2004); preprint arXiv:cond-mat/0401073.

2J. A. Elliott, "Novel approaches to multiscale modelling in materials science," Int. Mater. Rev. 56(4), 207-225 (2011).

${ }^{3}$ L. Chen, C. Wolverton, V. Vaithyanathan, and Z. Liu, "Modeling solid-state phase transformations and microstructure evolution,” MRS Bull. 26(3), 197-202 (2001).

${ }^{4}$ V. Vaithyanathan, C. Wolverton, and L. Chen, "Multiscale modeling of precipitate microstructure evolution," Phys. Rev. Lett. 88(12), 125503 (2002).

${ }^{\mathbf{5}} \mathrm{V}$. Vaithyanathan, C. Wolverton, and L. Chen, "Multiscale modeling of $\theta^{\prime}$ precipitation in Al-Cu binary alloys," Acta Mater. 52(10), 2973-2987 (2004).

${ }^{6}$ D. Molnar, R. Mukherjee, A. Choudhury, A. Mora, P. Binkele, M. Selzer, B. Nestler, and S. Schmauder, "Multiscale simulations on the coarsening of $\mathrm{Cu}$-rich precipitates in $\alpha$-Fe using kinetic Monte Carlo, molecular dynamics and phase-field simulations," Acta Mater. 60(20), 6961-6971 (2012).

${ }^{7}$ G. Demange, L. Lunéville, V. Pontikis, and D. Simeone, "Prediction of irradiation induced microstructures using a multiscale method coupling atomistic and phase field modeling: Application to the Ag-Cu model alloy,” J. Appl. Phys. 121(12), 125108 (2017).

${ }^{8}$ F. Soisson and G. Martin, "Monte Carlo simulations of the decomposition of metastable solid solutions: Transient and steady-state nucleation kinetics," Phys. Rev. B 62(1), 203 (2000).

${ }^{9}$ V. L. Ginzburg, V. L. Ginzburg, and L. D. Landau, Zh. Eksp. Teor. Fiz. 20, 1064 (1950).

${ }^{10}$ P. C. Hohenberg and B. I. Halperin, "Theory of dynamic critical phenomena," Rev. Mod. Phys. 49, 435-479 (1977).

${ }^{11} \mathrm{H}$. Emmerich, The Diffuse Interface Approach in Materials Science: Thermodynamic Concepts and Applications of Phase-Field Models (Springer Science \& Business Media, 2003), Vol. 73.

${ }^{12}$ J. A. Warren and B. T. Murray, "Ostwald ripening and coalescence of a binary alloy in two dimensions using a phase-field model," Modell. Simul. Mater. Sci. Eng. 4(2), 215 (1996).

${ }^{13}$ Y. Wen, J. Lill, S. Chen, and J. Simmons, "A ternary phase-field model incorporating commercial CALPHAD software and its application to precipitation in superalloys," Acta Mater. 58(3), 875-885 (2010).

${ }^{14}$ L.-Q. Chen, "Phase-field models for microstructure evolution," Annu. Rev. Mater. Res. 32(1), 113-140 (2002).

${ }^{15}$ Z. Erdélyi, M. Pasichnyy, V. Bezpalchuk, J. J. Tomán, B. Gajdics, and A. M. Gusak, "Stochastic kinetic mean field model," Comput. Phys. Commun. 204, 31-37 (2016),

${ }^{16}$ See http://skmf.eu for the source code and a tutorial of the SKMF model.

${ }^{17}$ N. Storozhuk, K. Sopiga, and A. Gusak, "Mean-field and quasi-phase-field models of nucleation and phase competition in reactive diffusion," Philos. Mag. 93(16), 1999-2012 (2013).

${ }^{18}$ G. Martin, “Atomic mobility in Cahns diffusion model," Phys. Rev. B 41(4), 2279 (1990)

${ }^{19}$ I. Vernyhora, D. Ledue, R. Patte, and H. Zapolsky, "Monte Carlo investigation of the correlation between magnetic and chemical ordering in NiFe alloys," J. Magn. Magn. Mater. 322(17), 2465-2470 (2010).

${ }^{20}$ A. Gusak and T. Zaporozhets, "Martins kinetic mean-field model revisitedFrequency noise approach versus Monte Carlo," Metallofiz. Noveishie Tekhnol. 40(11), 1415-1435 (2018).

${ }^{\mathbf{2 1}}$ A. Gusak, T. Zaporozhets, and N. Storozhuk, "Phase competition in solid-state reactive diffusion revisited-Stochastic kinetic mean-field approach," J. Chem. Phys. 150(17), 174109 (2019).

${ }^{22}$ G. Demange, S. Dépinoy, L. Lunéville, D. Simeone, and V. Pontikis, "Irradiation-based design of mechanically resistant microstructures tuned via multiscale phase-field modeling," Sci. Rep. 8(1), 10237 (2018).

${ }^{23}$ Z. Erdélyi, M. Sladecek, L.-M. Stadler, I. Zizak, G. A. Langer, M. Kis-Varga, D. L. Beke, and B. Sepiol, "Transient interface sharpening in miscible alloys," Science 306(5703), 1913-1915 (2004),

${ }^{24}$ Z. Erdélyi, I. A. Szabó, and D. L. Beke, "Interface sharpening instead of broadening by diffusion in ideal binary alloys," Phys. Rev. Lett. 89, 165901 (2002),

${ }^{\mathbf{2 5}}$ B. D. Gajdics, J. J. Tomán, and Z. Erdélyi, “Composition dependent gradient energy coefficient: How the asymmetric miscibility gap affects spinodal decomposition in Ag-Cu?," Calphad (to be published).

${ }^{26}$ P. R. Subramanian and J. H. Perepezko, "The Ag-Cu (silver-copper) system," J. Phase Equilibr. 14(1), 62-75 (1993).

${ }^{27}$ G. Demange, L. Lunéville, V. Pontikis, and D. Simeone, "Prediction of irradiation induced microstructures using a multiscale method coupling atomistic and phase field modeling: Application to the Ag-Cu model alloy,” J. Appl. Phys. 121(12), 125108 (2017).

${ }^{28}$ D. B. Butrymowicz, J. R. Manning, and M. E. Read, "Diffusion in copper and copper alloys, part II. Copper-silver and copper-gold systems," J. Phys. Chem. Ref. Data 3(2), 527-602 (1974).

${ }^{29}$ J. W. Cahn, “On spinodal decomposition,” Acta Metall. 9(9), 795 (1961).

${ }^{30}$ V. L. Ginzburg and L. D. Landau, J. Exp. Theor. phys. USSR 20, 1064 (1950).

${ }^{31}$ G. Demange, M. Chamaillard, H. Zapolsky, M. Lavrskyi, A. Vaugeois, L. Lunéville, D. Simeone, and R. Patte, "Generalization of the Fourier-spectral 
Eyre scheme for the phase-field equations: Application to self-assembly dynamics in materials," Comput. Mater. Sci. 144, 11-22 (2018).

${ }^{32}$ G. Radnóczi, E. Bokányi, Z. Erdélyi, and F. Misják, "Size dependent spinodal decomposition in Cu-Ag nanoparticles," Acta Mater. 123, 82-89 (2017).

${ }^{33}$ W. P. Davey, "Precision measurements of the lattice constants of twelve common metals," Phys. Rev. 25, 753-761 (1925),

${ }^{34}$ R. Ghez, Diffusion Phenomena: Cases and Studies (Springer 2010).

${ }^{35}$ Y. Adda, J. Philibert, and V. Pontikis, "Validity of the diffusion equation at the atomic scale investigated via numerical simulations," Phys. Rev. B 85, 144121 (2012),

${ }^{36}$ Z. Erdélyi, D. L. Beke, P. Nemes, and G. A. Langer, "On the range of validity of the continuum approach for nonlinear diffusional mixing of multilayers," Philos. Mag. A 79(8), 1757-1768 (1999),

${ }^{37}$ Z. Erdélyi and D. L. Beke, "Importance of proper choice of transition rates in kinetic simulations of dynamic processes," Phys. Rev. B 70, 245428 (2004),
${ }^{38}$ C. Wagner, "Theory of precipitate change by redissolution," Z. Elektrochem. 65, 581-591 (1961).

${ }^{39}$ I. M. Lifshitz, "The kinetics of precipitation from supersaturated solid solutions," J. Phys. Chem. Solids 19(1-2), 35-50 (1961).

${ }^{40}$ A. Ardell, "The effect of volume fraction on particle coarsening: Theoretical considerations," Acta Metall. 20(1), 61-71 (1972).

${ }^{41} \mathrm{~A}$. Baldan, "Review progress in Ostwald ripening theories and their applications to nickel-base superalloys part I: Ostwald ripening theories," J. Mater. Sci. 37(11), 2171-2202 (2002).

${ }^{42} \mathrm{P}$. Streitenberger, "Analytical description of phase coarsening at high volume fractions," Acta Mater. 61(13), 5026-5035 (2013).

${ }^{43}$ V. Vaithyanathan and L. Chen, "Coarsening of ordered intermetallic precipitates with coherency stress," Acta Mater. 50(16), 4061-4073 (2002).

${ }^{44}$ T. Wang, G. Sheng, Z.-K. Liu, and L.-Q. Chen, "Coarsening kinetics of $\gamma^{\prime}$ precipitates in the Ni-Al-Mo system,” Acta Mater. 56(19), 5544-5551 (2008). 